\title{
PROYECTO DE RESTAURACIÓN DE LOS TALUDES Y ZONAS ANEJAS A UNA AUTOVÍA
}

\author{
(RESTAURATION PROJECT OF HIGHWAY ROADBANKS AND SURROUNDING \\ AREAS )
}

Ignacio Claver Farias, Dr. Ingeniero de Montes. Catedrático de la U.P.M.

Manuel Díaz Martín, Licenciado en Ciencias Biológicas.

Master en Evaluación y Corrección de Impactos Ambientales.

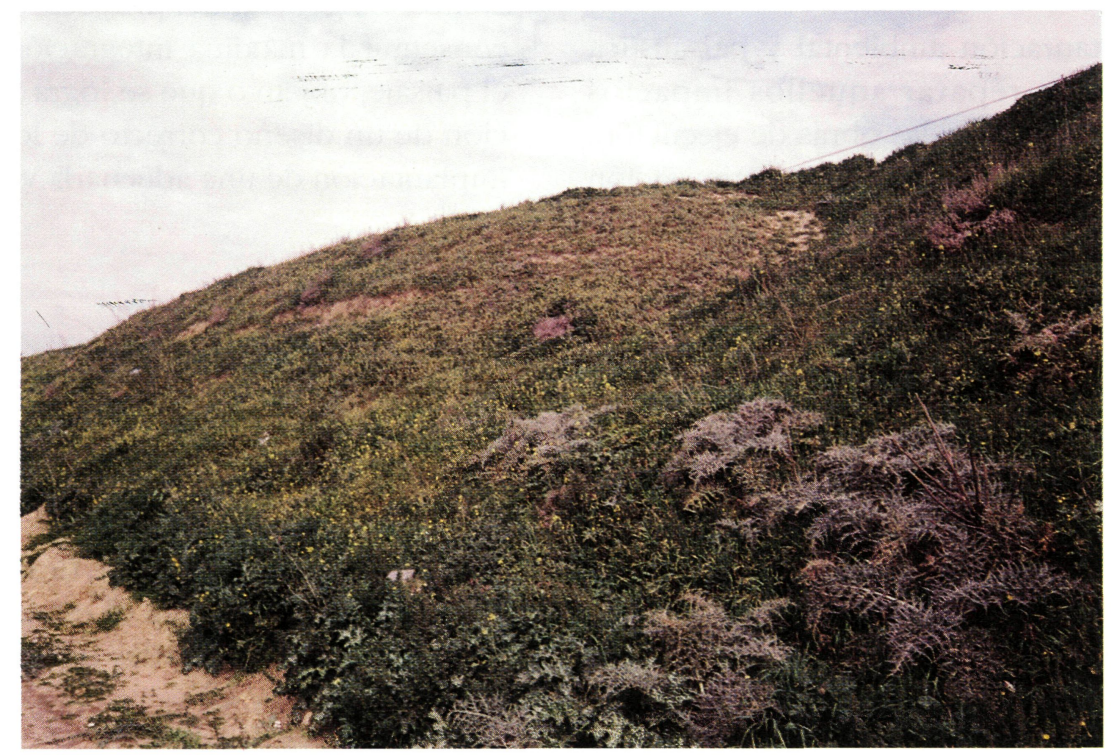

Tratamiento de taludes

\section{RESUMEN}

El propósito de este articulo es el de dar una visión general sobre los aspectos más importantes a considerar en la restauración ambiental de las zonas afectadas por la construcción de una autovía. El primer aspecto a considerar en toda restauración es la morfología resultante de la obra, comprobar si ésta es la adecuada, y si no es así, proceder a remodelar esta superficie con el objeto de conseguir un relieve lo más acorde con el entorno; se intentará de igual forma tender hacia la máxima estabilidad. A continuación, es conveniente mejorar el sustrato con el fin de favorecer el arraigo de los vegetales a implantar.

Por último, ha de procederse a la implantación vegetal, siendo importante la elección de especies a utilizar y el diseño empleado.

\section{SUMMARY}

The aim of this article is to give a general view of the most important aspects to be taken into account in the environmental restauration of areas affected by the construction of a highway. The first aspect to be considered in the whole restauration is the morphology resulting from the work, which should be checked for adequacy. In case it proves inadequate the surface should be remodelled in order to achieve a relief as much in accordance with the surroundings as possible. Maximum stability should also be pursued. Further on, it is convenient to improve the substratum to favour the plants' rooting.

Finally, plants should be introduced, taking care of the selection of the species and the design used. 


\section{INTRODUCCIÓN}

En la última década se ha producido un aumento de la preocupación por todo aquello que rodea al medio ambiente, observándose un cambio en el pensamiento ante la incidencia de las actividades humanas sobre el medio. Estas actividades transforman el espacio donde se realizan, planteándose así una gran problemática acerca de la adecuación ambiental de las actuaciones humanas.

Sin duda, una de las actividades antrópicas que mayor incidencia tienen en el entorno son las infraestructuras de transporte, alterando el paisaje natural de modo casi irreversible. Las autovías constituyen uno de los tipos de infraestructura que más afecta y transforma el medio por sus características particulares de linealidad y dimensión, así como por su efecto barrera en el entorno.

El proyecto de restauración ambiental y paisajística de autovías pretende reparar aquellos impactos negativos producidos durante las obras de ejecución, dando las soluciones necesarias para la minimización de las alteraciones e integración paisajística. La ocultación o enmascaramiento de los efectos negativos se logra disminuyendo las vistas poco agradables mediante la introducción de barreras visuales vegetales. La integración de la autovía en el paisaje se consigue restaurando la vegetación con especies similares a las presentes en el entorno. En conjunto, las actuaciones proyectadas para la recuperación del entorno inciden de forma directa sobre el medio mejorando su perspectiva tanto para los usuarios de la autovía como para el resto de individuos.

\section{OBJETIVOS}

Los objetivos de un proyecto de restauración de una autovía son los de definir y diseñar las obras necesarias para estabilizar los taludes y recuperar las zonas anejas alteradas por su construcción, regenerando e integrando, en la medida de lo posible, el entorno de la obra en el paisaje, siempre con la mayor capacidad de automantenimiento posible.

Fundamentalmente, se persiguen una serie de objetivos específicos:

Análisis de todas las áreas afectadas por la obra o sus actuaciones complementarias, para la integración paisajística de la obra en su conjunto.

Remodelado de las formas del paisaje resultante, suavizando los bordes.
- Reposición en taludes y demás superficies afectadas del suelo (tierra vegetal) que se ha retirado y apilado convenientemente en una fase previa.

- Tratamiento y revegetación de los taludes y zonas anejas, medianas, áreas comprendidas entre los ramales de los enlaces, vertederos, zonas de localización de instalaciones auxiliares, zonas de préstamo de materiales, viales empleados para el movimiento de maquinaria, etc.

- Enmascaramiento y ocultación de aquellas zonas afectadas que sean más visibles.

- Disminución de las molestias producidas a personas: contaminación atmosférica, sónica, etc.

- Establecimiento de un Plan de mantenimiento.

- La filosofía general del proyecto de recuperación es conseguir la máxima integración de la autovía en el paisaje, objetivo que se logra mediante la aplicación de un diseño correcto de los tratamientos y la implantación de una adecuada vegetación.

\section{ESTUDIO DE LOS ELEMENTOS DEL MEDIO}

La descripción de los elementos del medio donde se construye la autovía nos permite conocer las características del entorno donde se desarrolla el proyecto, aspecto fundamental para el establecimiento de más medidas correctoras acordes con las condiciones particulares de cada zona.

Previamente a la recogida de información de los distintos elementos del medio biofísico que van a constituir el inventario, es preciso definir la escala de trabajo, es decir, el nivel de detalle a utilizar en la cartografía de los mismos; normalmente éste depende de la fase de trabajo en la que nos encontremos, que en el caso de proyecto suele ser 1/1000 ó 1/500.

Los elementos del medio que deben inventariarse en cualquier caso para un conocimiento adecuado del medio donde se desarrolla una autovía son: clima, geología y geomorfología, suelos, vegetación, fauna y paisaje.

Otros elementos tales como agua, secursos culturales, procesos, etc. se describirán en caso necesario en función de las exigencias y características especificas del entorno del proyecto. 
El clima condiciona la estructuración de los ecosistemas, ya que determina en alto grado el tipo de suelo y vegetación, e influye en los usos del suelo; se puede decir que la descripción del clima nos sirve de información básica para la interpretación de otros aspectos del medio.

La caracterización climática puede hacerse mediante los valores registrados en las estaciones meteorológicas que se encuentran próximas a la zona donde se localizará la autovía. Podemos considerar los siguientes parámetros: temperatura, precipitación, período de heladas, ETP, viento, clasificación climática, etc. Dentro de estos parámetros es importante considerar sus valores extremos, ya que éstos pueden ser definitorios en el desarrollo de las especies vegetales a implantar.

\section{GEOLOGÍA Y GEOMORFOLOGÍA}

El estudio de estos elementos nos puede ayudar a determinar la estabilidad de los taludes generados por la autovía o la capacidad portante de los materiales existentes. Esta información nos permite además una mejor interpretación de otros elementos del medio como pueden ser el suelo, la vegetación o la hidrología.

Deben estudiarse aspectos como la morfología de la traza de la autovía y las características geológicas del entorno, con el objeto de determinar los posibles procesos y riesgos que puedan desencadenarse en torno al proyecto. Un factor importante es la exposición general de la infraestructura y la específica de los terraplenes y desmontes, con objeto de determinar la mayor o menor insolación, factor importante a considerar en la selección adecuada de especies vegetales.

\section{SUELOS}

El estudio del suelo nos permite determinar las características del sistema donde se va a desarrollar la recuperación. La estructura del suelo es importante para un buen desarrollo de las plantas, ya que de ella depende la cantidad de nutrientes. El tamaño y distribución de las partículas que lo forman condicionan otros factores como humedad, temperatura o aireación que son determinantes en la germinación de las semillas y desarrollo de los vegetales.

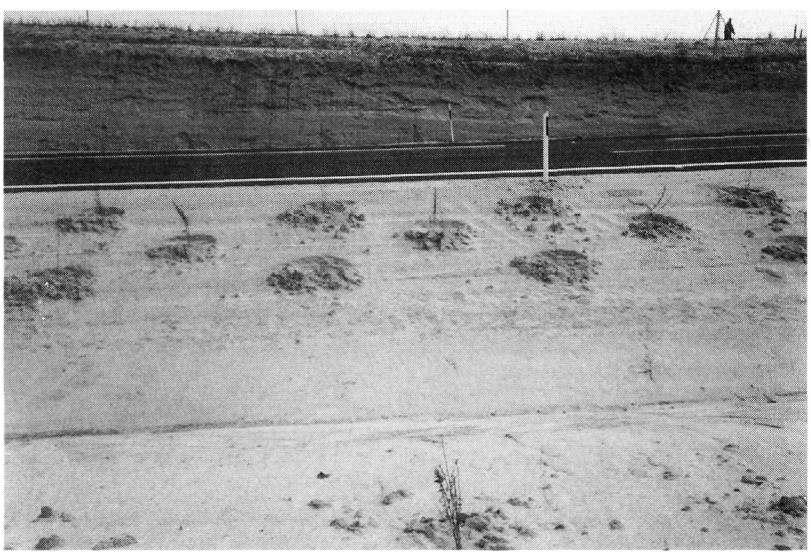

Plantaciones en mediana

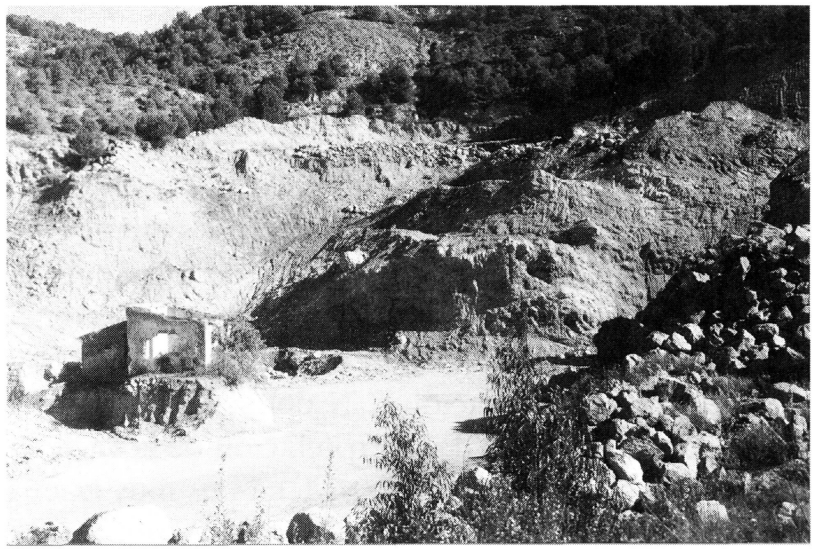

Zona de préstamo

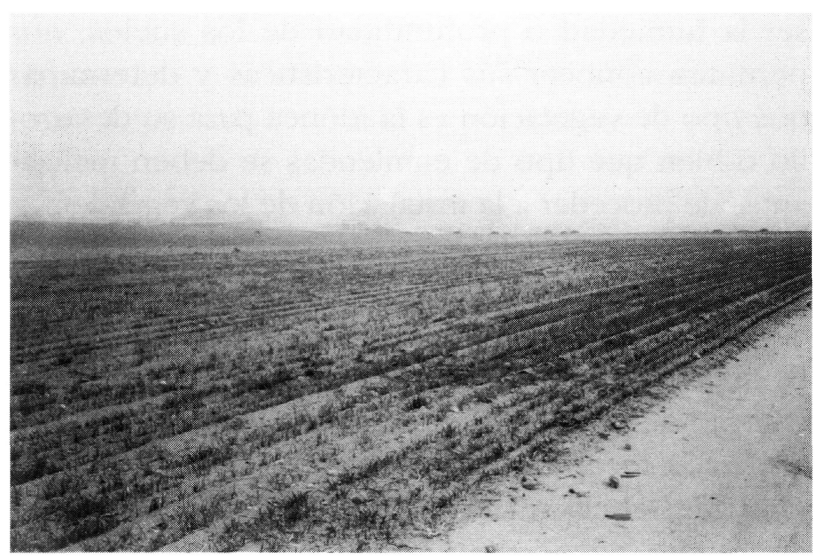

Restauración en zona de préstamo 

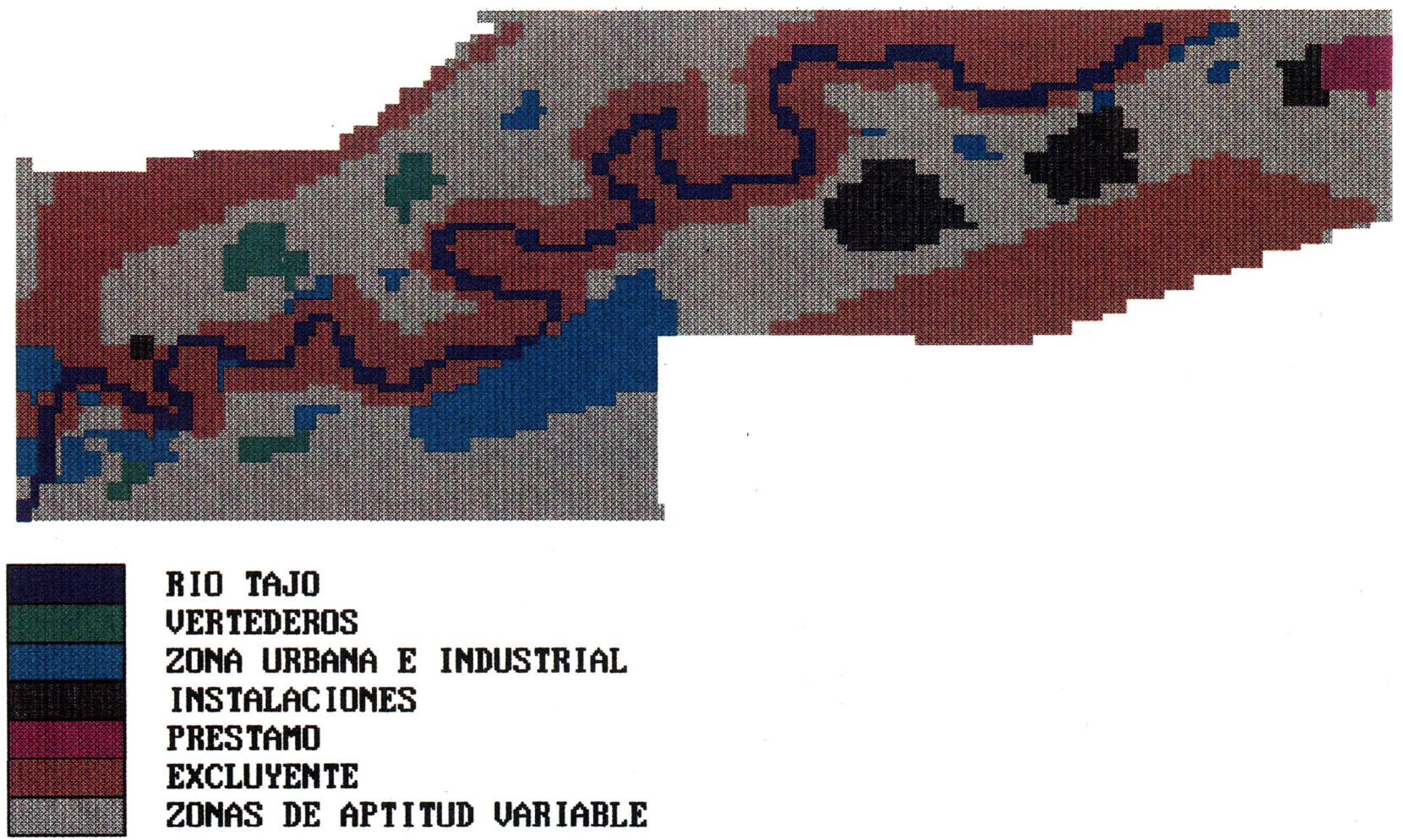

\section{RIO TAJO \\ UERTEDEROS \\ ZONA URBANA E INDUSTRIAL \\ INSTALAC IONES \\ PRESTAMO \\ EXCLUYENTE \\ ZONAS DE APTITUD UARIABLE}

Los suelos arenosos o areno-arcillosos presentan mejores condiciones para un rápido desarrollo de la vegetación que los suelos arcillosos duros. El contenido en materia orgánica mejora las condiciones físicas del mismo; la relación $\mathrm{C} / \mathrm{N}$ del suelo puede ser utilizada como índice de interpretación de la materia orgánica. Así crea relación C/N, 1:1,14 es muy buena y 1:1,66 es muy mala (NICOLAS 1968).

La absorción de nutrientes está íntimamente ligada al pH del suelo; así cuando sus valores son bajos se pueden producir pérdidas de nutrientes por lavado, mientras que si son muy altos pueden existir dificultades a la hora de absoberlos.

Todos estos factores junto con otros, como pueden ser la humedad o profundidad de los suelos, nos permiten conocer sus características y determinar qué tipo de vegetación es la idónea para su desarrollo o bien qué tipo de enmiendas se deben realizar antes de proceder a la instalación de los vegetales.

\section{VEGETACIÓN}

Es importante conocer la vegetación existente en el entorno de la autovía, con el fin de mostrar una pauta para la recuperación ambiental e integración paisajística. Del mismo modo se debe potenciar la zona de estudio, conociendo así las plantas que se desarrollan de forma natural en el entorno.
El estudio de vegetación conviene hacerlo considerando comunidades vegetales, existiendo varios métodos basados en diferentes criterios de clasificación. A partir de aquí se puede determinar qué especie o especies son dominantes, así como cuál es el espectro de formas biológicas que se desarrollan, datos a considerar a la hora de crear modelos de plantación que se asemejen a la vegetación existente en la zona.

\section{FAUNA}

Los aspectos que deben considerarse en el estudio de fauna son el inventario faunístico de las especies presentes en la zona de estudio y el hábitat donde se desarrollan estas especies. Fundamentalmente debe enfocarse el estudio hacia aquellas especies amenazadas o en peligro de extinción y a aquellas otras que puedan verse afectadas gravemente con la ejecución del proyecto. El objetivo específico que debemos atender es el de conseguir recuperar o restaurar sus hábitats naturales.

\section{PAISAJE}

El paisaje es considerado en la actualidad como un elemento del medio más comparable al resto, aunque en el fondo deba ser entendido como algo más complejo que alberga toda una serie de características del medio físico. 
Dos deben ser los aspectos más destacados a considerar en el estudio del paisaje; la visibilidad y la calidad. Es importante realizar un análisis del paisaje mediante el cálculo de la cuenca visual, sin hacer una valoración intrínseca del paisaje, cuestión ésta propia de los estudios previos al proyecto.

La recuperación ambiental no debe reducir la cuenca visual desde el interior de la autovía, y debe orientarse a la integración de la obra en el medio, procurando no producir contraste de forma, color, textura, etc. en el entorno próximo.

\section{MEDIDAS CORRECTORAS Y PROTECTORAS}

Los tratamientos de recuperación ambiental están encaminados a eliminar, en el mejor de los casos, o reducir los efectos negativos generados en el medio por la construcción de la autovía. Hay que tener en cuenta que un buen diseño del proyecto de obra es fundamental para el adecuado desarrollo de los tratamientos a aplicar.

Los objetivos fundamentales de estas medidas son solucionar los problemas creados por la obra, es decir estabilización de los taludes, recuperación de los suelos e integración paisajística, en definitiva, minimización de los impactos.

Los criterios utilizados para la elaboración de los tratamientos de recuperación han de ser ambientales, con el objeto de acercarse lo máximo posible al paisaje del entorno, intentando camuflar de una forma eficaz las obras de infraestructura y disminuyendo los posibles impactos en el medio.

A la hora de diseñar las medidas correctoras hay que tener en cuenta otros factores correspondientes a la propia infraestructura, tales como la correcta visibilidad dentro de la autovía, la seguridad, la funcionalidad de las obras del proyecto, etc.

Un aspecto fundamental en el proyecto de recuperación es la extracción y acopio de la tierra vegetal de la zona afectada directamente por la autovía; es importante considerar la capa de tierra vegetal que existe en la zona por donde pasará la traza y que es necesario remover por necesidades de obra, debiendo retirarla y conservarla adecuadamente para su posterior utilización en las labores de recuperación ambiental. Una vez que la obra esté en condiciones de ir admitiendo la tierra vegetal, ésta comenzará a disponerse en las zonas que se vayan a restaurar, ya sean taludes, medianas, áreas entre ramales de enlaces, vertederos de materiales sobrantes de obra, etc.

Los criterios utilizados en la selección de especies vegetales juegan también un importante papel en el buen desarrollo de las medidas a aplicar; los más importantes a considerar son:

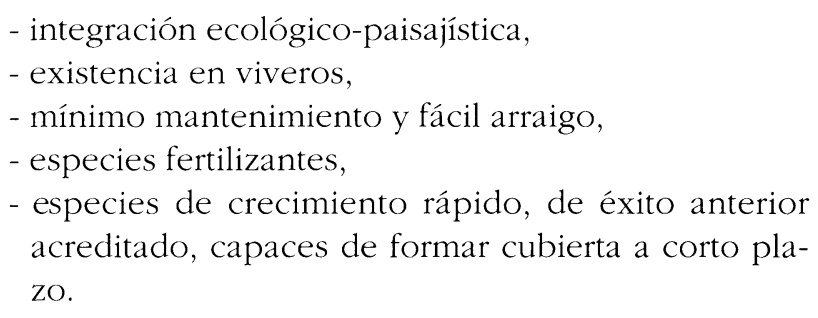

\section{TALUDES}

La aparición de desmontes y terraplenes en las autovías españolas es algo normal dado la topografía accidentada de nuestro territorio y los condicionantes específicos de la propia carretera. Estas estructuras llevan asociados una serie de problemas como pueden ser la inestabilidad, erosión, ruptura del paisaje, etc., de ahí que se apliquen tratamientos o medidas para minimizar estos efectos negativos, debiéndose buscar la integración visual y paisajística.

Los taludes son superficies con características poco idóneas para la instalación de vegetación de forma natural, así los desmontes suelen carecer de suelo para el desarrollo de las plantas al estar excavados normalmente en roca; los terraplenes, por el contrario, aglutinan una fuerte compactación con unas características de materiales prácticamente inertes. La aplicación de medidas correctoras y protectoras ayuda a la instalación de una cubierta vegetal en el talud con la disminución en procesos y riesgos que ello implica.

Uno de los principales factores en el desarrollo de los taludes es su diseño, así juegan un papel fundamental la pendiente y la altura de los mismos, existiendo inclinaciones por encima de las cuales es prácticamente imposible la instalación de vegetales; así pues, debe tenderse hacia la realización de taludes poco inclinados que disminuyen los efectos negativos del mismo y favorecen la aplicación y desarrollo de las medidas correctoras. En cuanto a la altura debe ser lo más pequeña posible ${ }_{2} \mathrm{y}$ en el caso de taludes de grandes dimensiones se dispondrán bermas o terrazas con objeto de disminuir los deslizamientos y escorrentía superficiales, y favorecer la 


\section{TRATAMIENTO P D. Plantación de arbustos en desmonte ( 1 planta/ $\mathbf{m}^{2}$ )}

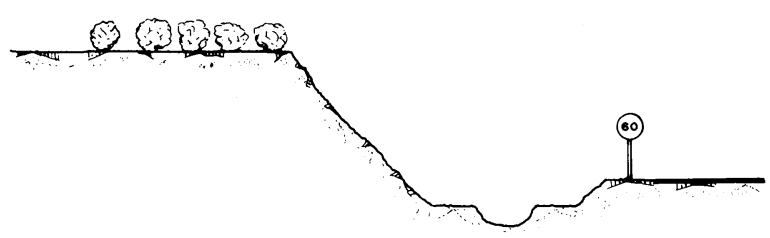

ALZADO
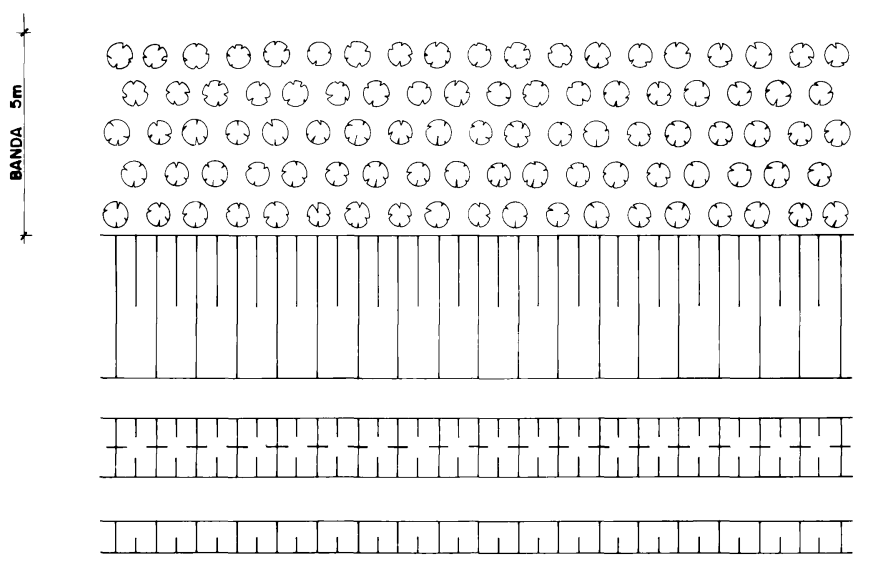

instalación de una cubierta vegetal. Por otra parte, también hay que tener en cuenta que los taludes con forma cóncava son los más estables, sometidos a una menor erosión y cambio en su perfil.

Las medidas de restauración aplicables en los taludes pueden ser muy variables. Un tratamiento bastante general a aplicar a la mayoría de ellos, puede ser el que se expone a continuación. En primer lugar debe llevarse a cabo un extendido de la tierra vegetal (retirada en la fase previa a las obras) sobre la superficie de los taludes, procurando que el reparto sea homogéneo, con el fin de mejorar el sustrato donde se van a instalar las plantas. A continuación, se realiza una hidrosiembra, técnica de siembra consistente en la proyección sobre el terreno de una mezcla de semillas, mulch, abonos, aditivos y agua, mediante una maquina hidrosembradora; la mezcla de semillas debe estar compuesta generalmente por gramíneas, leguminosas y subarbustivas seleccionadas en función del clima, suelo, vegetación del entorno y las características de las propias especies, así como su facilidad para conseguirlas. Una vez depositada la semilla en los taludes, es conveniente realizar un tapado de las mismas con objeto de protegerla de las condiciones adversas y evitar la degradación de los otros componentes de la hidrosiembra. La principal función de este tratamiento es la de reducir la erosión y estabilizar el terreno, y en la medida de lo posible disminuir el impacto visual y lograr una integración en el paisaje. El grado de protección con respecto a la erosión será directamente proporcional al poder tapizante de la mezcla de semillas seleccionadas.

También pueden llevarse a cabo plantaciones en el propio talud, aunque esta medida sólo se emplea en algunas ocasiones. Donde sí se suelen hacer plantaciones es en la cabecera y/o pie de talud, describiendo unas bandas de distintas dimensiones donde se plantan árboles o arbustos. Un aspecto fundamental en la integración en el entorno de estas plantaciones es su diseño, debiéndose huir de las disposiciones lineales que dirijan las vistas, ya que no se pretende hacer un parque, sino una implantación vegetal en un medio rural. El diseño debe respetar el carácter natural de la zona, y la elección de especies debe apoyarse sobre todo en las especies típicas del lugar. 

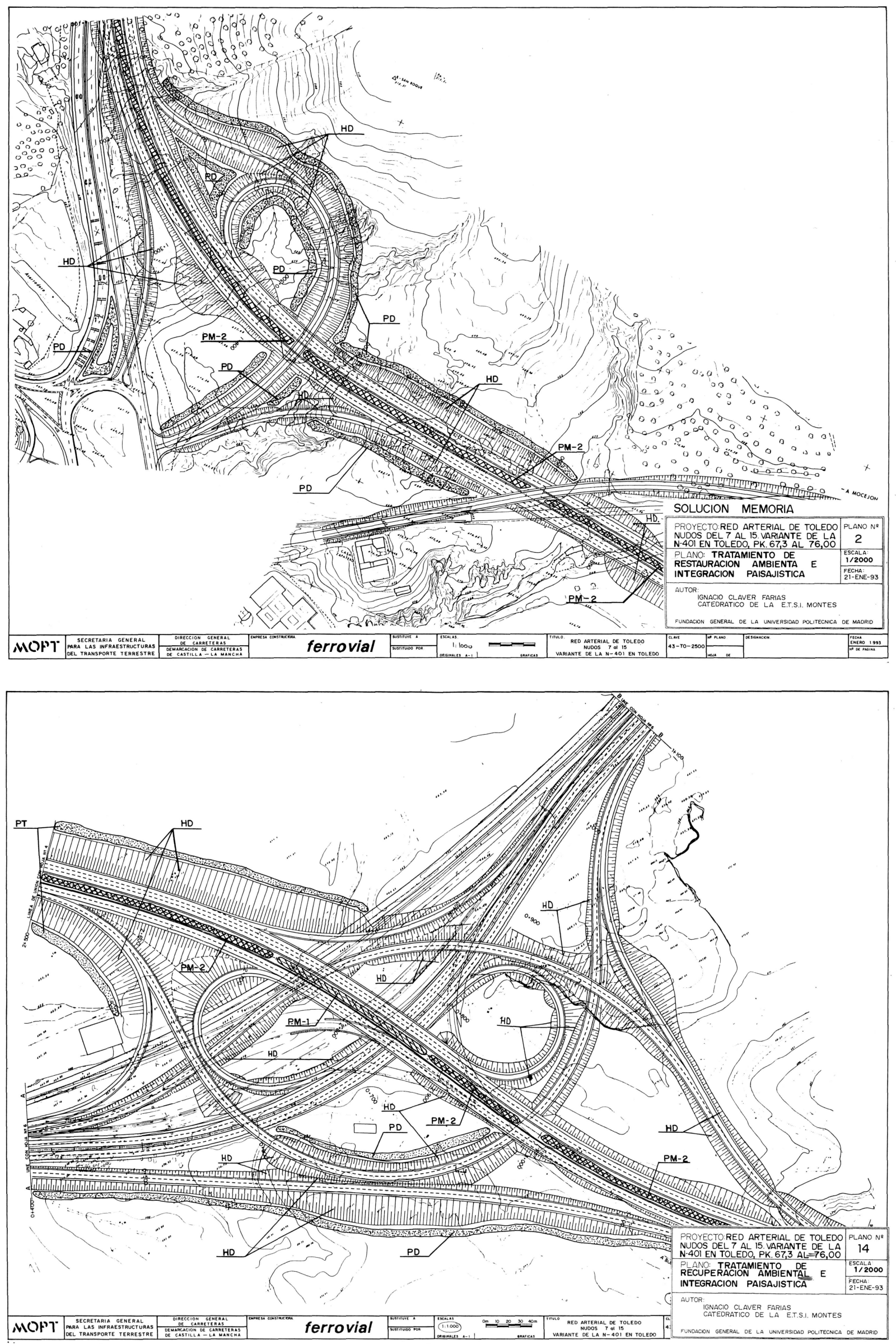
Las plantaciones consiguen una integración de la obra en el paisaje, y además evitan la presencia de cultivos en la banda más próxima a la traza, dada la posible afección por elementos contaminantes procedentes de los vehículos. Además, consiguen una protección del suelo, evitan los deslumbramientos, protegen contra la insolación, el viento, etc.

Las medidas de recuperación de los taludes basadas en la revegetación deben aplicarse lo antes posible en el tiempo, evitando así la formación de regueros en su superficie.

Existen otras técnicas para el tratamiento de taludes como pueden ser la disposición de mantas orgánicas, gunitados, muros prefabricados, gaviones, sistemas que cumplen diferentes objetivos, y que suelen aplicarse cuando las condiciones del talud son muy concretas.

\section{ZONAS AFECTADAS}

Las zonas anejas a una autovía pueden ser muy diversas en función de la propia infraestructura, aunque existe una serie de ellas comunes, entre las que podemos considerar medianas, áreas comprendidas entre los ramales de los enlaces, zonas de préstamo de materiales, zonas de vertedero de materiales sobrantes, zonas de instalaciones auxiliares, etc.

La mediana entre calzadas suele tener una anchura variable, definida principalmente en base a estudios económicos y funcionales, considerando parámetros como posibilidad de accidentes, precio de construcción de la plataforma, etc. Las medidas de recuperación en las medianas suelen consistir en una mejora del sustrato y, en una posterior revegetación, en función de la zona que atraviese; cuando son demasiado estrechas, estas medidas no son viables, y suele recurrirse a tratamientos denominados duros.

La mejora del sustrato consiste en una descompactación de la superficie y el posterior extendido de la tierra vegetal procedente de la retirada en una fase previa. El objeto de esta mejora es favorecer la supervivencia de la vegetación que se va a implantar. La revegetación propiamente dicha dependerá del tramo atravesado, aunque la práctica habitual es realizar una siembra y una posterior plantación de árboles y arbustos, siempre en función del espacio disponible. Uno de los objetivos fundamentales de estos tratamientos es el facilitar la conducción, disminuyendo los deslumbramientos entre vehículos y favoreciendo el seguimiento óptico de la carretera.
Las áreas comprendidas entre los ramales de los enlaces son superficies que quedan aisladas entre las distintas vías y que son muy visibles tanto desde dentro de la propia infraestructura como desde el exterior. Por lo general, los tratamientos aplicados a estas zonas consisten en el extendido de tierra vegetal por toda su superficie para mejorar el sustrato, y la posterior siembra y plantación. Las plantaciones se distribuirán teniendo en cuenta no entorpecer la visibilidad, debiéndose utilizar líneas de arbustos que guien el tráfico o árboles que, por su altura, no disminuyan las vistas para una buena conducción. Los grupos de plantación irán lo suficientemente separados de la calzada principal, para que en ningún caso resulten peligrosos sus fustes en el supuesto de que un vehículo se salga de la vía. Los distintos grupos, tanto arbóreos como arbustivos, deben dar la sensación de asociaciones vegetales naturales, para lo que no sólo se debe hacer una correcta selección de especies, sino también un diseño adecuado y coherente con el medio que lo rodea.

Las zonas de préstamo de materiales aparecen ligadas a todas las autovías, ya que es necesario disponer de distintos tipos de materiales tanto para la creación de pasos elevados o terraplenes, como para la propia construcción de la calzada. Lo idóneo sería aprovechar la graveras que existen en el entorno de la autovía para extraer los materiales; esto no siempre es posible por no existir graveras, o bien por estar demasiado distantes de la traza, por lo que es preciso localizar zonas para la extracción de material.

La recuperación de estas zonas pasa como en la mayor parte de los casos por una remodelación de las formas del terreno, suavizando las aristas y una mejora del sustrato donde se va a realizar la implantación vegetal. En una fase previa se debe proceder a retirar la capa de suelo que exista para, una vez retirados los áridos, depositarla de nuevo sobre la superficie a restaurar. Previamente a la extensión del suelo debe descompactarse el terreno mediante un escarificado. El diseño de la recuperación dependerá fundamentalmente del entorno en el que nos encontremos; así si estamos en una zona de cultivos herbáceos los tratamientos consistirán en la siembra de las especies presentes, con el fin de devolver al territorio sus usos vocacionales. En el caso de ser zonas menos antropizadas, el tratamiento irá encaminado a una integración de la zona afectada en el paisaje mediante siembras y plantaciones de los árboles y arbustos presentes en el entorno, utilizando diseños similares a los naturales. 


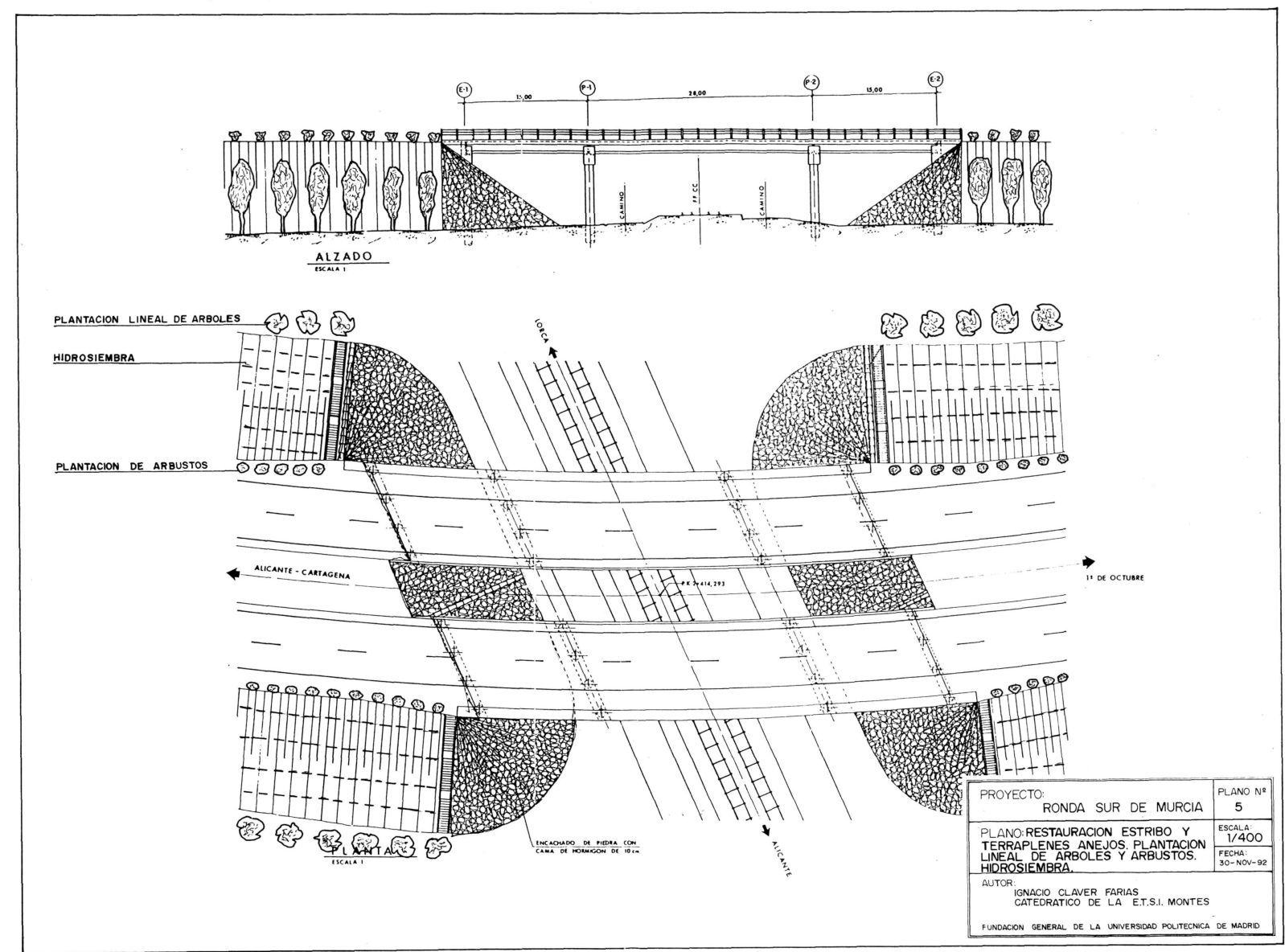

En el caso de las zonas destinadas a vertedero de materiales sobrantes de la obra, los tratamientos serán similares a los que se apliquen en las zonas de préstamo. La principal diferencia es la morfología resultante, ya que en un caso se crean huecos, y en el otro normalmente se tapan oquedades o se generan grandes acopios.

Como ya se ha mencionado en los casos anteriores, la primera fase del tratamiento consiste en la excavación de la capa superior de suelo de toda la superficie, que será tapada para cubrir posteriormente el relleno y mejorar las condiciones del sustrato. Posteriormente se aplicarán las medidas correctoras en función de su entorno inmediato, procediendo a la realización de siembras o plantaciones de árboles y arbustos.

La siembra, técnica de incorporación de semillas al suelo, puede hacerse a voleo requiriendo personal cualificado o por medio de una sembradora. Una vez incorporadas las semillas al suelo, es recomendable pasar un rastrillo de forma suave y extender una ligera capa de mantillo, con el fin de que éstas queden enterradas. El momento más indicado para realizar la siembra es durante el otoño y la primavera, en días sin viento y con suelo poco o nada húmedo.

Las zonas donde se localizan las instalaciones auxiliares son por lo general pequeñas extensiones con una morfología bastante llana. La restauración de estas zonas comienza con una descompactación del terreno, ya que han sido sometidas a un continuo circular de maquinarias pesadas; más tarde se procede a la mejora del sustrato existente, bien mediante la incorporación de tierra vegetal o mediante el uso de enmiendas y abonos, para posteriormente realizar la implantación vegetal.

En el caso de realizar plantaciones, debe prepararse el terreno con cuidado y manejar las plantas con delicadeza, con el fin de no dañar su parte aérea ni su parte radical.

Cuando la plantación no pueda realizarse en el momento de recibir las plantas en la obra, se procederá a su depósito en una zanja cubrierrdo las raíces con un mínimo de $20 \mathrm{~cm}$ de tierra adecuada, con la suficiente humedad y protegidas contra las heladas. 
Una vez realizada la plantación es conveniente hacer un alcorque cuyas dimensiones dependerán del tamaño de la planta.

Un aspecto que debe considerarse específicamente en el proyecto de construcción de la autovía (en caso de no haber sido tratado en el E.I.A.) es la localización de zonas de préstamo y/o vertedero, zonas de instalaciones auxiliares, etc. Debe realizarse en base a criterios ambientales con objeto de minimizar el impacto ambiental producido. Para ello pueden utilizarse modelos basados en la definición de elementos significativos y técnicas automáticas de tratamiento de la información.

\section{MANTENIMIENTO}

El mantenimiento es uno de los aspectos que deben considerarse específicamente en el proyecto de restauración de una autovía.

El objetivo del mantenimiento es favorecer el desarrollo de los vegetales y evitar que se mueran en los primeros años de vida, no dejando a la naturaleza la exclusividad de sacar adelante las revegetaciones diseñadas en el proyecto de restauración. Además hay que tener en cuenta que no todas las plantaciones tienen un objetivo estético e integrador, sino que también cumplen otras funciones, y deben someterse a unas labores de conservación para no entorpecer el tráfico y disminuir la seguridad vial.

Una buena selección de especies vegetales puede disminuir enormemente el mantenimiento, con el ahorro económico que esto puede suponer.

El mantenimiento debe comprender todas aquellas labores que se realizarán de forma periódica sobre las zonas revegetadas, para permitir el correcto desarrollo y evolución de las plantas tal y como se ha definido en el proyecto, y alcanzar así las características funcionales que las definen.

Las labores que más habitualmente suelen realizarse para la conservación de los tratamientos diseñados son las siguientes:

\section{- Riegos.}

- Siegas.

- Resiembras y reposición de marras.
- Podas.

- Abonados.

Los riegos deben hacerse en función de las características edafoclimáticas de la zona y en los meses con déficit hídrico; se ejecutarán en aquellas horas del día en las que la evapotranspiración sea mínima, es decir, a primera hora de la mañana o última de la tarde. El objetivo de esta labor es que los vegetales encuentren en el suelo, en todo momento, la cantidad de agua necesaria para su normal desarrollo.

Las siegas deben realizarse en aquellas superficies cubiertas de herbáceas anuales o vivaces con una densidad de población superior a la normal, que impide el correcto desempeño de las funciones marcadas. El periodo de siega por lo general es en primavera o en otoño.

Los restos procedentes de esta labor deberán ser recogidos y transportados a un lugar adecuado.

En aquellas zonas en las que se hayan realizado siembras y éstas no obtengan unos resultados de nascencia adecuados, deberá realizarse una resiembra con el fin de que toda la superficie tenga un reparto homogéneo de las especies vegetales sembradas.

De la misma forma cuando los árboles o arbustos mueran, deberán reponerse de tal forma que no comiencen a perderse unidades del diseño inicial y éste quede incompleto, y por tanto no cumpla las funciones para las que ha sido proyectado.

Las podas de las plantaciones pueden ser de dos tipos, poda de formación, realizada en especies jóvenes para dirigirles la forma, realizada para mantener los portes de las especies e impedir que éstas se desarrollen en exceso y causen molestias a los usuarios de la autovía. Los restos deberán ser transportados a un lugar adecuado. Se realizarán con la frecuencia necesaria para mantener un estado sanitario adecuado y una forma acorde con los objetivos para los que se han plantado.

Por último, conviene abonar aquellas superficies que han sido restauradas con el objeto de mejorar el sustrato y favorecer el desarrollo de los vegetales hasta que éstos hayan alcanzado u estado de madurez adecuado. Los abonados se realizarán durante la primavera o el otoño. 


\section{CONSIDERACIONES FINALES}

A lo largo de este trabajo hemos tratado de dar al proyectista los objetivos y aspectos más relevantes que se deben considerar en el proyecto de restauración de los taludes y zonas anejas a una autovía, los cuales a nuestro juicio proporcionan la información básica para poder redactar eficazmente la memoria de un proyecto de esta naturaleza. Es preciso integrar además otras determinaciones (generales y específicas o particulares) que configurarán la documentación del proyecto y que a título orientativo podemos resumir como sigue:

\section{DOCUMENTO № 1. MEMORIA Y ANEJOS}

\section{MEMORIA}

1. Antecedentes,

2. Objeto del Proyecto.

3. Descripción del proyecto.

4. Factores y condicionantes ambientales.

5. Justificación de la solución adoptada.

6. Mediciones.

7. Plazo de ejecución y garantía.

8. Fórmula de revisión de precios.

9. Clasificación del contratista.

10. Presupuestos.
ANEJOS
1. Inventario ambiental
2. Elección de especies.
3. Plan de obras.
4. Justificación de precios.

5. Plan de mantenimiento.

6. Proyecto de Seguridad e Higiene (en su caso).

\section{DOCUMENTO № 2. PLANOS}

\section{DOCUMENTO № 3. PLIEGO DE CONDICIONES}

1. Introducción.

2. Descripción de las obras.

3. Materiales.

4. Ejecución de las obras.

5. Medición y abono.

6. Obligaciones del contratista.

7. Condiciones generales.

\section{DOCUMENTO № 4. PRESUPUESTO}

1. Mediciones.

2. Cuadro de precios $n^{\circ} 1$.

3. Cuadro de precios no 2 .

4. Presupuestos.

Deseamos que la lectura de este artículo facilite a Ingenieros y Constructores la difícil labor de conseguir restauraciones de calidad que contribuyan a integrar la autovía en el paisaje. A este respecto la realización de proyectos de restauración por equipos multidisciplinares, especialistas en los diversos factores ambientales, dará credibilidad y garantizará los objetivos propuestos. 